\title{
Widely Tunable Silicon-On-Insulator Ring Resonators with a Liquid Crystal Cladding
}

\author{
Wout De Cort ${ }^{* \dagger \ddagger}$, Jeroen Beeckman ${ }^{\dagger \ddagger}$, Tom Claes ${ }^{* \ddagger}$, Kristiaan Neyts ${ }^{\dagger \ddagger}$ and Roel Baets ${ }^{* \ddagger}$ \\ *Photonics Research Group, Dept. Information Technology, Ghent University - IMEC, Belgium \\ ${ }^{\dagger}$ Liquid Crystals and Photonics Group, Dept. Electronics and Information Systems, Ghent University, Belgium \\ ${ }^{\ddagger}$ Center for Nano- and Biophotonics (NB-Photonics), Ghent University \\ Email: wout.decort@ugent.be
}

\begin{abstract}
Silicon-On-Insulator ring resonators can be widely tuned with a cladding layer of Liquid Crystals. Reorientation of the director with an electric field results in a tuning range of more than $30 \mathrm{~nm}$.
\end{abstract}

\section{INTRODUCTION}

Ring resonators are efficient optical filters designed to filter out narrow wavelength bands from a broad spectrum. This characteristic makes them very useful in larger telecommunication components such as (de-)multiplexers. In some applications, e.g. reconfigurable optical networks, it is necessary to be able to adjust the position of the resonances. Another reason why tunablity is desirable is that current fabrication techniques can not yet guarantee an exact resonance wavelength. This tunability in ring resonators is often achieved by heating or carrier injection. These methods provide a considerable but still limited tuning range. They require a constant current and thus a constant power consumption. Tuning with a liquid crystal (LC) cladding layer is another method which is not widely used but which has been investigated in the past. Nematic LC is a very attractive material for this purpose due to its high anisotropy $(\Delta \mathrm{n} \approx 0.2)$. For ring resonators guiding TE-polarized (Transverse Electric field) light it has been shown that with a top-bottom electrode configuration, the resonances can be shifted towards shorter wavelengths [1]. The field generated by electrodes in a finger pattern can shift the resonance towards longer wavelengths [2]. The mechanics of tuning with an LC cladding are becoming clear but the tuning ranges achieved are still below $1 \mathrm{~nm}$ [1], [2], [3] or require complicated fabrication techniques [4]. In this work we show that TM (Transverse Magnetic field) ring resonators in SiliconOn-Insulator (SOI) can be tuned over a wide range $(>30 \mathrm{~nm})$ with a straightforward top-bottom electrode configuration. This is to the best of our knowledge the highest tuning range reported for silicon-based resonators and opens opportunities for widely tunable filters, lasers and detectors.

\section{OVERVIEW OF THE CELL}

\section{A. Cell structure}

The base of the cell consists of an SOI chip. This material system is widely used in electronics and integrated optics and its fabrication processes are well-known. The waveguides are

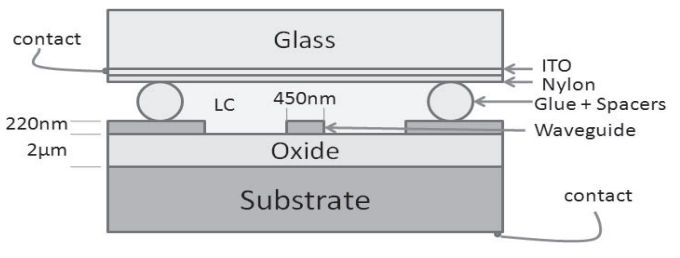

Fig. 1. Schematic overview of a liquid crystal cell.

defined in a $220 \mathrm{~nm}$ thin $\mathrm{Si}$ layer which is separated from the $\mathrm{Si}$ substrate by an insulating $\mathrm{SiO}_{2}$ layer to prevent optical leakage [5]. With glue and spacers ( $6 \mu \mathrm{m}$ diameter) we attach a glass plate to the chip. Near the gap between the glass plate and the chip we deposit a drop of E7 (a commercially available nematic LC often used in displays) which is sucked into the cell by capillary forces. On the glass plate there are two additional layers. First, a thin Indium Tin Oxide (ITO) layer for electrical contacting. Second, a spincoated alignment layer (nylon) to give the LC director a fixed orientation near the surface of the glass plate. For easy contacting, electrical wires are soldered to the ITO layer and to the substrate which acts as the bottom contact.

\section{B. Ring resonators}

The waveguides measure $220 \mathrm{~nm}$ high and $450 \mathrm{~nm}$ wide. The ring resonators have a bend radius of $20 \mu \mathrm{m}$ and 4 straight sections of $10 \mu \mathrm{m}$ each. This adds up to a total length of $166 \mu \mathrm{m}$. The coupling section is $4 \mu \mathrm{m}$ long and the gap between the bus waveguide and the resonator is $550 \mathrm{~nm}$ wide. The bus waveguide is equipped with two grating couplers. These allow light to be coupled vertically into and out of the waveguide [6].

\section{WORKING PRINCIPLES AND SIMULATIONS}

On SOI surfaces, the LC aligns in a planar fashion. The director is able to follow the structures on the chip down to the nanometer scale [1]. In case of ring resonators, the director will remain parallel with the ring waveguide in the area near the waveguide due to geometrical alignment. The TM mode travelling in the waveguide has evanescent tails protruding from the top and bottom of the waveguide into the cladding layers. As the electric field is mostly polarized along the normal to the surface, it will see the ordinary refractive 


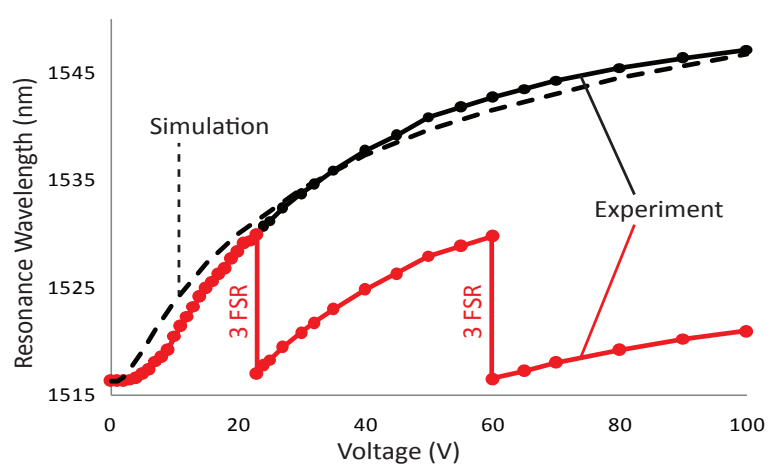

Fig. 2. The resonance wavelength as a function of voltage over the cell. Simulation (dashed), the actual measured curve (below) and the total accumulated shift (black) are shown.

index $\left(\mathrm{n}_{o}\right)$ of the LC. This results in a low value of the effective index of the mode. When an electric field is applied between the silicon substrate and the ITO on the glass plate, the director will reorient vertically. Depending on the strength of the field, the evanescent tails of the TM mode will now see more of the extraordinary refractive index $\left(\mathrm{n}_{e}\right)$ of the LC and the effective index will rise. The change in effective index translates directly to a change in resonance wavelength of the ring resonator.

$$
\frac{\Delta \lambda}{\lambda}=\frac{\Delta n_{e f f}}{n_{g}},
$$

where $\mathrm{n}_{g}$ is the group index. We simulate the wavelength shift by combining a calculation of the director with a fully anisotropic mode solver. The results of the director calculation are imported in the mode solver to get a detailed view on the behavior of the cell at different voltages. The simulated tuning curve for the waveguides specified above shows a tuning range of around $31 \mathrm{~nm}$ for a voltage of $100 \mathrm{~V}$ (Fig. 2). There is a threshold voltage around $5 \mathrm{~V}$ where the field exactly compensates for the elastic forces holding the LC in place.

\section{CHARACTERISATION}

Light from a tunable laser is coupled into the waveguide through the grating couplers and the output spectrum is analyzed. To avoid ion drift which can cause shielding of the electrodes, we apply a $10 \mathrm{kHz}$ square wave to the LC cladding layer. For increasing voltage levels the resonance shifts towards longer wavelengths (Fig. 3). Note that at certain voltage levels we start tracking resonance dips located 3 free spectral ranges towards shorter wavelengths. The measured curve as well as the accumulated shift is shown in Fig. 2. The agreement between simulation and experiment is excellent. At $10 \mathrm{~V} \mathrm{rms}$ the resonance wavelength has shifted about $4 \mathrm{~nm}$ which covers a free spectral range in the rings used for this experiment. When we increase the voltage to $100 \mathrm{~V}$ rms we can shift the resonance up to $31 \mathrm{~nm}$. At this point the resonance does not change significantly with increasing voltage as the director has reached its maximum tilt angle throughout the cell.

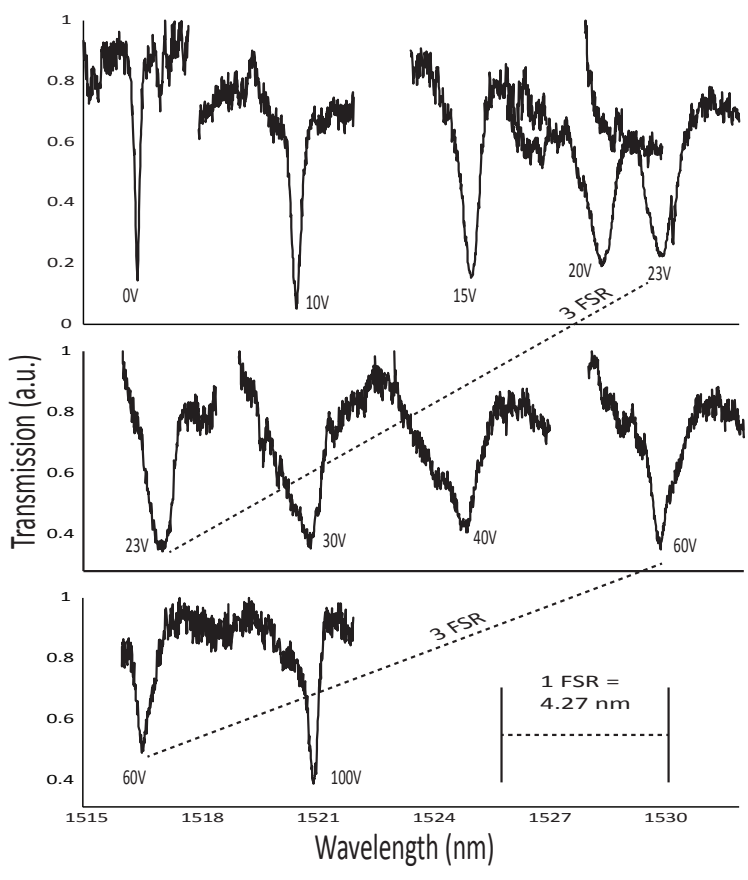

Fig. 3. Output spectrum for different values of the voltage.

\section{CONCLUSION}

We have shown that TM ring resonators on SOI can be tuned widely with a cladding layer of LC. A tuning range of more than $30 \mathrm{~nm}$ is achieved. We have explained the tuning mechanism and shown simulations that correspond well with the experimental findings. These results have a large potential for widely tunable filters, lasers and detectors, with low tuning power.

\section{ACKNOWLEDGMENT}

W. De Cort acknowledges the Institute for the Promotion of Innovation through Science and Technology in Flanders (IWTVlaanderen) for a specialization grant. The work has been carried out in the framework of the IAP project Photonics@ be of the Belgian Science Policy.

\section{REFERENCES}

[1] W. De Cort, J. Beeckman, R. James, F. Fernandez, R. Baets, and K. Neyts, "Tuning of silicon-on-insulator ring resonators with liquid crystal cladding using the longitudinal field component," Optics Letters, vol. 34, no. 13, pp. 2054-2056, 2009.

[2] _ " "Tuning silicon-on-insulator ring resonators with in-plane switching liquid crystals," Journal of the Optical Society of America B-Optical Physics, vol. 28, no. 1, pp. 79-85, 2011.

[3] B. Maune, R. Lawson, C. Gunn, A. Scherer, and L. Dalton, "Electrically tunable ring resonators incorporating nematic liquid crystals as cladding layers," Applied Physics Letters, vol. 83, no. 23, pp. 4689-4691, 2003.

[4] V. G. Chigrinov, L. Zhou, A. Muravsky, and A. W. O. Poon, "Electrically tunable microresonators using photoaligned liquid crystals," U.S. Patent 20070258677 , November 8, 2007.

[5] S. Selvaraja, P. Jaenen, W. Bogaerts, D. Van Thourhout, P. Dumon, and R. Baets, "Fabrication of photonic wire and crystal circuits in siliconon-insulator using $193 \mathrm{~nm}$ optical lithography," Journal of Lightwave Technology, vol. 27, no. 18, p. 4076 4083, 2009.

[6] D. Taillaert, P. Bienstman, and R. Baets, "Compact efficient broadband grating coupler for silicon-on-insulator waveguides," Optics Letters, vol. 29, no. 23, pp. 2749-2751, 2004. 